\title{
Coping Mediates the Association Between Type D Personality and Perceived Health in Chinese Patients with Coronary Heart Disease
}

\author{
Xiao-nan Yu • Zhansheng Chen • Jianxin Zhang • \\ Xiaohui Liu \\ Published online: 13 October 2010 \\ (C) The Author(s) 2010. This article is published with open access at Springerlink.com
}

\begin{abstract}
Background Increasing evidence show that Type D personality is a risk factor for morbidity, mortality, and quality of life of patients with coronary vascular disease. Few studies examined coping as a potential behavioral mechanism to explain the harmful effect of Type D personality.

Purpose This study examined the association between Type D personality, coping, and perceived health among Chinese patients with coronary heart disease (CHD).

Methods One hundred seventeen CHD patients completed the assessments on Type D personality, coping, perceived severity of CHD, and morale.

Results There was no difference on severity of coronary artery stenosis between Type D and non-Type D patients. Compared to the non-Type D patients, the Type D patients perceived higher severity of CHD (5.31 \pm 2.41 versus $4.45 \pm$ $2.17, p<0.05)$ and lower morale $(12.67 \pm 4.71$ versus $15.00 \pm$ $4.43, p<0.05)$, and used less confrontation $(16.90 \pm 5.39$ versus $20.88 \pm 4.95, p<0.001)$ and more acceptance-resigna-
\end{abstract}

X.-n. Yu $(\bowtie)$

School of Public Health, Faculty of Medicine,

The University of Hong Kong,

Pokfulam, Hong Kong

e-mail: yuxn@hku.hk

Z. Chen

Department of Psychology, The University of Hong Kong,

Pokfulam, Hong Kong

J. Zhang

Institute of Psychology, Chinese Academy of Sciences,

Beijing, People's Republic of China

X. Liu

Cardiology Department, Beijing Anzhen Hospital,

Capital University of Medical Sciences,

Beijing, People's Republic of China tion coping (10.16 \pm 3.50 versus $8.35 \pm 3.48, p<0.05)$. Mediation analyses showed that confrontation coping mediated the association between Type D personality and perceived severity of disease, and acceptance-resignation coping mediated the association between Type D personality and morale after controlling for age, gender, and clinical variables.

Conclusion The Type D patients used maladaptive coping in response to disease. These coping strategies fully mediated the association between Type D personality and perceived health. Implications for integrating coping training into the intervention for patients with a Type $\mathrm{D}$ personality are discussed.

Keywords Coping - Morale - Perceived severity of disease . Type D personality

\section{Introduction}

Type D personality refers to the synergistic tendency of experiencing negative emotions (negative affectivity, NA) and inhibiting emotional expression in social interactions (social inhibition, SI) [1]. Accumulating evidence has shown that Type D personality predicts morbidity and mortality of coronary vascular disease (CVD), and impairs the quality of life and subjective well-being of CVD patients [2].

Understanding of the mechanisms through which Type $\mathrm{D}$ takes effect is scarce. Some efforts have been made to examine the Type D mechanisms, including the physiological markers such as cardiovascular reactivity [3], cortisol dysregulation [4], proinflammatory cytokine [5, 6], and behavioral mechanisms of an unhealthy lifestyle (e.g., skipping a regular medical checkup) [7]. Further studies on psychosocial mechanisms are warranted. 
Individuals use various styles to adapt to chronic illness. Coping in a medical context refers to the cognitive and behavioral efforts to manage the disease. Self-blame, avoidance, and social isolation were more prominent among the patients with coronary heart disease (CHD), compared to the general population [8]. The effects of coping on psychological adjustment have been investigated, for example, approach coping enhanced subjective wellbeing and decreased negative emotion of the CHD patients [9]. Active confrontational coping predicted decreased clinical progression of the disease [10]. Nevertheless, there is scarce systematic knowledge with regard to coping of the Type D patients. They may employ maladaptive coping in face of a health threat.

Coping not only directly affects the psychological adjustment to disease, but also mitigates the association between personality and adaptation, for instance, denial and confrontation mediated the association between optimistic personality and distress among women with breast cancer [11]. Therefore, it is reasonable to assume a mediating model of coping to explain the link between Type D personality and psychological adjustment.

At present, little is known about the association between Type D personality and perceived health in Chinese patients with CHD. A few Chinese studies showed that Type D is associated with higher depression and anxiety $[12,13]$ and lower quality of life [14]. However, no report is available on the association between Type D personality and perceived severity of illness in Chinese culture. In the present study, we investigated CHD patients' perception about severity of illness. Perceived health can predict allcause and cardiac death after controlling for risk factors, severity of disease, psychosocial factors, and demographic characteristics [15]. Poor perceived physical health status is associated with adverse prognosis in patients with CVD [16]. Previous study showed that Type D patients reported more symptoms than non-Type D patients [7]. However, it is unknown whether Type D patients would perceive their disease more severe than non-Type D patients.

In addition, to assess psychological well-being of the elderly people, the present study measured morale. With components of satisfaction with self and positive attitudes toward aging, morale is used to indicate quality of life for the old population. For the patients with chronic heart failure, those with higher morale reported better social functioning regardless of the severity of disease [17]. The Philadelphia Geriatric Center Morale Scale, which provides a multidimensional approach to assess psychological wellbeing, has been recommended as one of the standardized measurements for old persons by the United Kingdom's Royal College of Physicians and the British Geriatric Society [18]. Attitude toward aging, a subscale of the Philadelphia Geriatric Center Morale Scale, was one of the best predictors of mortality after controlling for sociodemographic characteristics, self-rated health, and number of illness [19]. With self-rated severity of CHD and morale, this study examined general perception of health in physical and psychosocial perspectives.

The aims of this study are to examine the perceived health of patients with a Type D personality, their coping reactions in the medical context, and the potential route of coping to link Type D personality and perceived health in a Chinese sample. The hypotheses of this study are as follows:

1) Type D patients would perceive higher severity of CHD and lower morale compared to the non-Type D patients;

2) The patients with a Type D personality would use less adaptive coping styles and more maladaptive coping than the other patients;

3) The adaptive coping styles would be associated with lower self-rated severity of disease and higher morale, and the maladaptive coping styles would work reversely; and

4) The association between Type D personality and perceived health would be mediated by coping after controlling for demographic and clinical variables.

Figure 1 illustrates the hypothesized mediating effect of coping between Type D personality and perceived health.

\section{Methods}

\section{Participants}

The participants consisted of 117 consecutive patients who were diagnosed with CHD. They were hospitalized for percutaneous coronary intervention (PCI) in the Beijing Anzhen Hospital, the Capital University of Medical Sciences, and the Beijing Institute of Heart Lung and Blood Vessel Disease from September to December of 2005. Patients were excluded from this study if they had comorbidities of life-threatening disease (e.g., cancer, leucocythemia), psychiatric disorder (e.g., schizophrenia), or cognitive disability (e.g., dementia). Few patients with myocardial infarction were admitted to the study division, so we excluded such patients in order to control for the confounding factors. The participants averaged $59 \pm 8.0$ years of age (ranging from 40 to 78 years) of whom $29.9 \%$ were females. The response rate was $77.5 \%$. The responders and non-responders had no significant differences on demographic and clinical characteristics ( $p>0.05$, results not reported).

Participants were recruited during their hospitalization for PCI. The physicians introduced the study to the patients and referred them to the interviewer (the first author). All of 


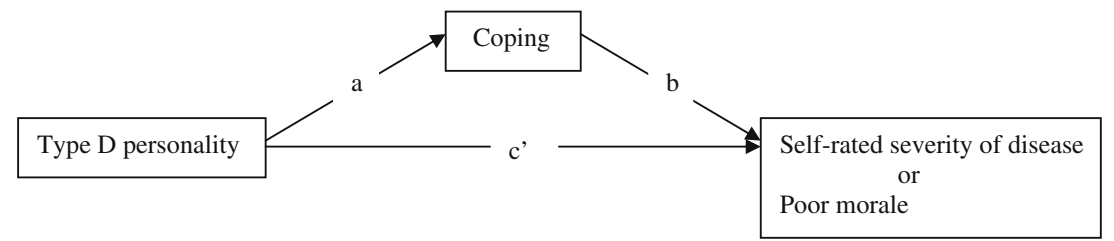

Fig. 1 Graphic representation of the mediation model. Type D personality affects self-rated severity of disease or morale indirectly through coping. The total effect $(c)$ is composed of a direct effect $\left(c^{\prime}\right)$ and an indirect effect $(a \times b)$

the patients were informed that the service they receive would not be affected if they refuse to join the study. Informed written consents were obtained from all participants. Face-to-face interviews were carried out in a private setting before PCI. Medical data (e.g., coronary artery stenosis) were derived from the patients' medical charts under their permissions. The data collection process was described in detail in another published paper that was based on the same dataset [13]. Only 117 patients were included in this study since their medical records of coronary artery stenosis were available. Ethics approval was obtained from the Institute of Psychology, Chinese Academy of Sciences.

\section{Measurements}

Demographic information The information of gender and age were collected.

Clinical variables Smoking, family history of CVD, hypertension, and diabetes mellitus were recorded. The degree of coronary artery stenosis was assessed by coronary angiography using a Gensini scoring system [20]. High scores indicate higher severity of coronary artery stenosis.

Type D personality The Type D Scale measures NA (e.g., "I often feel unhappy") and SI (e.g., "I feel inhibited in social interactions") [21]. A five-point Likert scale from 0 (false) to 4 (true) was used. The cut-off of $\geq 10$ on both subscales was used to define cases with a Type D personality. The internal reliability coefficients were 0.88 and 0.86 for the NA and SI subscales, respectively [21]. For the Chinese version, the Cronbach's $\alpha$ coefficients of the NA and SI subscales were 0.90 and 0.85 [13]. In this study, the Cronbach's $\alpha$ coefficients of the NA and SI subscales were 0.89 and 0.87 , respectively.

Coping Coping strategies were assessed by the Medical Coping Modes Questionnaire [22]. It includes 19 items and three subscales: confrontation (e.g., "ask for advice from the health professionals"), avoidance (e.g., "change subjects whenever others talk about the disease"), and acceptanceresignation (e.g., "do nothing about the illness"). For the confrontation, avoidance, and acceptance-resignation sub- scales, the Cronbach's $\alpha$ coefficients were $0.70,0.66$, and 0.67 , respectively. For the Chinese version, the internal consistency coefficients were $0.69,0.60$, and 0.76 [23]. The response rating ranged from "never" to "very much" with a four-point Likert scale. The Cronbach's $\alpha$ coefficients of the three subscales in the present study were $0.76,0.74$, and 0.66 , respectively.

Perceived severity of CHD It was assessed by the cardiovascular disease part in the Cornell Medical Index Health Questionnaire [24]. The item of "Doctor said you have heart trouble" was deleted because all patients were diagnosed with $\mathrm{CHD}$; the item regarding family history was deleted because this question was included in the previous part. The remaining 11 items in the cardiovascular part include severity of high blood pressure, chest pain, and breathing difficulty. The participants rated their condition in the past 6 months on a yes/no format (yes $=1$, no $=0$ ). Higher scores indicate higher severity. The scores of the Chinese version could predict those who remained healthy and those who died or incurred a major illness in a longitudinal study [25].

Morale Morale was assessed by the Philadelphia Geriatric Center Morale Scale [26]. Questions included "Little things bother me more this year" and "I am as happy as I was when I was younger." Higher scores indicate higher morale. Good internal reliability of the scale was reported [26]. The Chinese version showed internal reliability coefficient as 0.86 [27]. The Cronbach's $\alpha$ coefficient was 0.83 in this study.

\section{Statistical Analyses}

The differences on demographic characteristics and clinical variables of Type D and non-Type D patients were compared with the Chi-square test (Fisher's exact test when appropriate) or Student's $t$ test. Student's $t$ test was used to compare the differences on perceived severity of disease, morale, and coping between two groups. The effect size of differences (Cohen's $d$ ) on coping was calculated. An effect size of 0.20 was regarded as small, 0.50 as moderate, and 0.80 as large [28]. Pearson correlation analysis was applied to examine the association between coping and dependent variables. To test the indirect effects of Type $\mathrm{D}$ personality via 
the proposed mediating variables of coping on self-rated severity of CHD and morale, we performed a Sobel test using a bootstrapping method with 5,000 bootstrap resamples [29]. Using a point estimate of the mediation effect with a $95 \%$ confidence interval, bootstrapping suggests significant mediation effect ( $p<0.05$, two-tailed) when zero is not contained in the $95 \%$ confidence interval. Thus, the effect of Type D personality on dependent variable (self-rated severity of CHD or morale) would be mediated by the mediating variables such as confrontation. SPSS for Windows version 14.0 was performed for statistical analysis, and $p<0.05$ was considered statistically significant.

\section{Results}

Demographic Information and Clinical Variables

Table 1 summarizes the patient characteristics. The prevalence of Type D in this sample was $31.4 \%$. There were no significant differences between the Type D patients and non-Type $\mathrm{D}$ patients on gender, severity of coronary artery stenosis, family history of CVD, hypertension, diabetes mellitus, or smoking. However, the Type D patients were younger than the non-Type $\mathrm{D}$ patients $(56.71 \pm 9.21$ versus $61.43 \pm 10.40, p<0.05)$.

Type D Personality and Perceived Health Status

The Type D patients self-rated the disease more severe $(5.31 \pm 2.41$ versus $4.45 \pm 2.17, p<0.05)$ and perceived lower morale $(12.67 \pm 4.71$ versus $15.00 \pm 4.43, p<0.05)$ compared to the non-Type $\mathrm{D}$ patients.

Type D Personality and Coping

The Type D patients used less confrontation (16.90 \pm 5.39 versus 20.88 $\pm 4.95, p<0.001$; Cohen's $d=0.77$ ) and more acceptance-resignation coping $(10.16 \pm 3.50$ versus $8.35 \pm$
3.48, $p<0.05$; Cohen's $d=0.52)$ than the non-Type D patients. There was no significant difference on avoidance between the two groups $(14.74 \pm 6.03$ versus $14.15 \pm 5.82$, $p>0.05$ ).

\section{Coping and Perceived Health}

Higher confrontation was significantly associated with lower perceived severity of CHD $(r=-0.28, p<0.01)$ and greater morale $(r=.25, p<0.05)$. Higher acceptanceresignation was significantly associated with lower morale $(r=-0.31, p<0.01)$ but not self-rated severity of CHD $(r=$ $0.07, p>0.05$ ). Avoidance was not significantly associated with perceived severity of CHD $(r=0.02, p>0.05)$ or morale $(r=-0.04, p>0.05)$.

Mediating Effect of Coping on the Association Between Type D and Perceived Health

The results of the mediation analyses are presented in Table 2. In the analysis to predict perceived severity of disease, Type $\mathrm{D}$ personality was the independent variable and confrontation was the potential mediator, with gender, age group, severity of CHD, family history of CVD, smoke, hypertension, and diabetes mellitus controlled. Type D personality was significantly associated with perceived severity of CHD (c) and confrontation (a). Confrontation was significantly associated with self-rated severity of CHD (b). The indirect effect for confrontation $(\mathrm{a} \times \mathrm{b})$ was estimated to lie between -0.68 and -0.04 . Because zero was not in the $95 \%$ confidence interval, we can conclude that the indirect effect was significantly different from zero at $p<0.05$, and confrontation mediated the relationship between Type D personality and self-rated severity of CHD after controlling for demographic and clinical variables. As the direct effect of Type D on self-rated severity of CHD (c') was not significant after confrontation entered the model, confrontation fully mediated the effect of Type D on self-rated severity of CHD (Table 2).

Table 1 Patient demographic characteristics and clinical variables

\begin{tabular}{|c|c|c|c|c|c|}
\hline & $\begin{array}{l}\text { Total sample }(n=117) \\
n(\%)\end{array}$ & $\begin{array}{l}\text { Type D }(n=37) \\
n(\%)\end{array}$ & $\begin{array}{l}\text { Non-Type D }(n=80) \\
n(\%)\end{array}$ & Chi-square & $P$ \\
\hline Male, $n$ & $82(70.1)$ & $25(67.6)$ & $57(71.3)$ & 0.26 & 0.87 \\
\hline Age $($ mean $\pm \mathrm{SD})$ & $59.26 \pm 8.02$ & $56.71 \pm 9.21$ & $61.43 \pm 10.40$ & $-2.14^{\mathrm{a}}$ & 0.02 \\
\hline Coronary artery stenosis (mean $\pm \mathrm{SD}$ ) & $37.20 \pm 33.64$ & $37.55 \pm 30.40$ & $37.13 \pm 32.76$ & $1.09^{\mathrm{a}}$ & 0.94 \\
\hline Family history of CVD & $59(50.4)$ & $19(51.4)$ & $40(50.0)$ & 0.21 & 0.65 \\
\hline Hypertension & $82(70.1)$ & $29(78.4)$ & $53(66.3)$ & 0.17 & 0.68 \\
\hline Diabetes mellitus & $21(17.9)$ & $6(16.2)$ & $15(18.8)$ & 0.12 & 0.91 \\
\hline Smoking & $53(45.3)$ & $16(43.2)$ & $37(46.3)$ & 0.12 & 0.91 \\
\hline
\end{tabular}

${ }^{a}$ Differences on continuous variables were compared with Student's $t$ test 
Table 2 Summary of mediation results for self-rated severity of CHD and morale

\begin{tabular}{|c|c|c|c|c|c|c|c|c|c|}
\hline $\begin{array}{l}\text { Dependent } \\
\text { variable (DV) }\end{array}$ & $\begin{array}{l}\text { Independent } \\
\text { variable (IV) }\end{array}$ & Mediator (M) & Variables controlled & $\begin{array}{l}\text { Effect of IV } \\
\text { on M (a) }\end{array}$ & $\begin{array}{l}\text { Effect of } \mathrm{M} \text { on } \\
\mathrm{DV}(\mathrm{b})\end{array}$ & $\begin{array}{l}\text { Direct } \\
\text { effects (c') }\end{array}$ & $\begin{array}{l}\text { Indirect } \\
\text { effects }(\mathrm{a} \times \mathrm{b})\end{array}$ & $\begin{array}{l}\text { Total } \\
\text { effects (c) }\end{array}$ & $\begin{array}{l}\text { Adjusted } R \\
\text { square }\end{array}$ \\
\hline $\begin{array}{l}\text { Self-rated } \\
\text { severity } \\
\text { of CHD }\end{array}$ & Type D & Confrontation & $\begin{array}{l}\text { Gender } \\
\text { Age } \\
\text { Severity of coronary } \\
\text { artery stenosis } \\
\text { Family history of } \\
\text { CVD } \\
\text { Smoke } \\
\text { Hypertension } \\
\text { Diabetes mellitus }\end{array}$ & $-5.49 * * *$ & $-0.09 *$ & 0.28 & $-0.23 *$ & $0.80^{*}$ & $0.18^{* *}$ \\
\hline Morale & Type D & $\begin{array}{l}\text { Confrontation } \\
\text { Acceptance-- } \\
\text { resignation }\end{array}$ & $\begin{array}{l}\text { Gender } \\
\text { Age } \\
\text { Severity of coronary } \\
\quad \text { artery stenosis } \\
\text { Family history of CHD } \\
\text { Smoke } \\
\text { Hypertension } \\
\text { Diabetes mellitus }\end{array}$ & $\begin{array}{c}-5.44 * * * \\
2.11 * *\end{array}$ & $\begin{array}{c}0.18^{*} \\
-0.50^{* *}\end{array}$ & 0.18 & $\begin{array}{l}-0.97 \\
-1.05^{*}\end{array}$ & $-1.83^{*}$ & $0.14 *$ \\
\hline
\end{tabular}

$*, \mathrm{p}<.05$

$* *, \mathrm{p}<.01$

$* * *, \mathrm{p}<.001$

In the analysis to predict morale, Type D personality was the independent variable, confrontation and acceptanceresignation were the potential mediators, and gender, age group, severity of CHD, family history of CVD, smoke, hypertension, and diabetes mellitus were controlled. Type D personality was significantly associated with morale (c) and two coping styles (confrontation and acceptance-resignation, a). These coping styles were both significantly associated with morale (b). The indirect effects for confrontation and acceptance-resignation $(\mathrm{a} \times \mathrm{b})$ were estimated to lie between -2.69 and 0.29 , and -2.63 and -0.15 , respectively. Because zero was not in the $95 \%$ confidence interval for acceptance-resignation, we can conclude that this direct effect is significantly different from zero at $p<0.05$, and acceptance-resignation mediated the relationship between Type D personality and morale after controlling for demographic and clinical variables. In addition, acceptance-resignation fully mediated the association above since Type D's direct effect (c') was not significant. However, the indirect effect of confrontation did not significantly differ in magnitude, thus confrontation did not contribute as a mediator in the effect of Type D on morale (Table 2).

\section{Discussion}

This is the first study to examine the association between Type D personality and perceived health in Eastern culture with a sample of Chinese patients suffering from CHD.
Given few research reporting the coping styles in response to the illness among the Type D population, this study provides new knowledge on the coping characteristics of Type D patients and coping route by which the Type D personality exerts harmful effect on psychological adjustment among CHD patients.

The present study showed that Type D personality could not be attributed to disease severity since the studied Type $\mathrm{D}$ and non-Type D patients had no difference on severity of coronary artery stenosis, which is in line with other studies in cardiac patients in Western samples [30], for example, a recent study showed that there are no differences on baseline left ventricular ejection fraction, comorbidity, previous myocardial infarction, coronary artery bypass graft, or PCI between patients with and without a Type D [31]. These results suggested that illness severity is not a risk factor for a Type D personality. The pattern might apply to both Western and Eastern population considering the prevalence of Type D is similar in different cultural contexts such as Belgium, Germany, Denmark, and China, ranging from $24 \%$ to $31 \%$ [13, 21, 32, 33]. More studies are needed to compare the prevalence of a Type D among patients with mild and severe disease across cultures.

An important finding in this study is that the Type D patients rated their disease as more severe than the nonType D ones although their degrees of coronary artery stenosis were similar. This study is one of the very first studies to report the association between Type D personality and poor perception of cardiovascular health status in the Eastern society. Research in the Western society also 
found that Type D patients report a poor health status which cannot be accounted for by indicators of disease severity such as left ventricular dysfunction. These results are consistent across studies and cardiovascular diagnosis (e.g., coronary artery disease, congestive heart failure, peripheral artery disease) and treatment (including PCI and implantable cardioverter-defibrillator therapy), as summarized in an overview on the impact of Type D on health status [34]. Our study provides new evidence with the Chinese sample that Type D personality rather than disease severity contributed to poor perception of cardiovascular health. It is interesting to examine whether the impact of Type D on health perception might be generalized to other cultural background.

Consistent with a wealth of data on Type D's risk effects on subjective well-being obtained in Western countries [2], this study showed that the Type D patients reported less morale than their counterparts. For patients with CHD, Type D personality strongly decreased their self-esteem and quality of life, and increased hopeless and depressive symptoms when these elderly people are threatened by chronic illness, impaired functioning, and grief. This finding makes sense since NA is one of the two components of a Type D personality [21], and depression is a predominant factor contributing to low morale among elder people [35]. Morale is an important indicator of psychosocial well-being of elderly people, and subjective health is more closely linked with morale rather than objective health [36]. The harmful effect of Type D personality on morale suggests that Type $\mathrm{D}$ might be a risk factor for positive aging of elderly people with chronic illness.

In the present study, as compared to their non-Type D counterparts, patients with a Type D personality were likely to use less confrontation, and more acceptance-resignation, in coping with their disease. It is worthy to note that the effect size of the difference on confrontation approached large. Our results are consistent with a UK study which showed that individuals with a Type D personality tend to use more maladaptive coping strategies such as resignation and withdrawal [37]. The poor coping of Type D patients might result from SI, another component of Type D personality. Previous studies showed that interpersonal inhibition was associated with poorer adherence to treatment [38], and social inhibited individuals were less likely to perform health-promoting behaviors such as physical activity [39]. In addition, the traditional Chinese culture of "feeling at ease whatever the circumstances" may contribute to the usage of acceptance-resignation coping among these Chinese patients. In our study, the Type D patients define the illness as stressful and out of their control, and in turn, they feel hopeless to change the reality. Therefore, the Type D patients tend to accept the illness as end of life rather than actively seek treatment and support. This finding has potential clinical implications since it may shed light on interpretation of the risk effect of Type D personality on poor adherence to treatment.

This study fills in the knowledge gap of coping as a behavioral vehicle between Type D personality and psychological adjustment. In this study, coping accounted for the overall effect of Type D personality on both physical health perception and psychological well-being. Type D patients tend to reduce the usage of confronting coping and are vulnerable to low perception of health status, while they employ acceptance-resignation, which in turn makes these patients suffer from low morale. These results on mediation effect of coping among Type D persons suggest that it would be promising to integrate coping skills training into self-management and psychological intervention for Type $\mathrm{D}$ patients. First, the coping intervention includes reappraising the disease attack as a challenge rather than a threat of life, reconstructing the meaning of the disease as an opportunity of growth, enhancing prospects of recovery, and building optimistic belief about adjustment to disease. Second, the patients could mobilize supportive resources (e. g., doctors, family, friends) to regulate negative emotions and get informational and tangible assistance. Furthermore, social network such as patient-support group supervised by experienced clinical psychologist might provide a safe environment to share experience and feeling for the Type D patients who have a common tendency of SI. Third, problem solving and confrontational coping in the medical context should be strengthened. The patients could inquire doctors about medical knowledge about CHD, methods to reduce pain, solutions to increase adherence, and approaches to make active involvement in treatment decision. Thus, the usage of maladaptive coping such as resignation and withdrawal would be reduced. Such a coping training in illness management would focus on empowerment of Type D patients to increase health control, reduce NA, improve coping self-efficacy and skills, and enhance their perceived health [40]. Although some Type D interventions (e.g., cognitive behavioral therapy, social skill training, stress management) were suggested to reduce stress and promote social ability, a coping intervention for Type D patients has not been suggested in previous studies.

The results of this study should be interpreted with caution. There are several limitations. First, the sample size is relatively small. Second, the measurements rely on selfreport. In addition, the acceptance-resignation coping subscale showed relatively low internal consistency (Cronbach's $\alpha=0.66$ ) although it was similar to that reported by Feifel et al. in the original study (Cronbach's $\alpha=0.67$ ) [22]. Third, the effects of neuroticism and extraversion were not controlled in this study. Type D 
personality has been criticized as a synonym for neuroticism and extraversion in the Big Five personality. However, some work showed that Type D personality was independently associated with posttraumatic stress disorder after adjusting for neuroticism and extraversion [32], and Type D personality was associated with fewer health behaviors and social support after controlling for neuroticism [7]. Fourth, the causal direction of Type D personality, coping, and psychological adjustment cannot be determined with the cross-sectional study design.

Overall, the findings of this study demonstrated the association between Type D personality and perceived health in a sample of Chinese CHD patients. The Type D patients rated their disease more severe, reported less morale, and used less confrontation and more acceptanceresignation coping compared to the non-Type D patients although two groups had similar severity of coronary artery stenosis. In addition, confrontation as well as acceptanceresignation coping played mediating roles in the association between Type D personality and perceived health. These findings indicate that more research is needed to integrate coping modification as a potential intervention among CHD patients with a Type D personality.

Open Access This article is distributed under the terms of the Creative Commons Attribution Noncommercial License which permits any noncommercial use, distribution, and reproduction in any medium, provided the original author(s) and source are credited.

\section{References}

1. Denollet J. Type D personality: a potential risk factor refined. J Psychosom Res. 2000;49:255-66.

2. Pedersen SS, Denollet J. Is Type D personality here to stay? Emerging evidence across cardiovascular disease patient groups. Curr Cardiol Rev. 2006;2:205-13.

3. Habra ME, Linden W, Anderson JC, Weinberg J. Type D personality is related to cardiovascular and neuroendocrine reactivity to acute stress. J Psychosom Res. 2003;55(3):235-45.

4. Whitehead DL, Perkins-Porras L, Strike PC, Magid K, Steptoe A. Cortisol awakening response is elevated in acute coronary syndrome patients with type-D personality. J Psychosom Res. 2007;62:419-25.

5. Denollet J, Conraads VM, Brutsaert DL, De Clerck LS, Stevens WJ, Vrints CJ. Cytokines and immune activation in systolic heart failure: the role of Type D personality. Brain Behav Immun. 2003;17:304-9.

6. Conraads VM, Denollet J, De Clerck LS, Stevens WJ, Bridts C, Vrints CJ. Type D personality is associated with increased levels of tumour necrosis factor (TNF)-alpha and TNF-alpha receptors in chronic heart failure. Int J Cardiol. 2006;113:34-8.

7. Williams L, O'Connor RC, Howard S, Hughes BM, Johnston DW, Hay JL, et al. Type-D personality mechanisms of effect: the role of health-related behavior and social support. J Psychosom Res. 2008;64:63-9.

8. Pignalberi C, Patti G, Chimenti C, Pasceri V, Maseri A. Role of different determinants of psychological distress in acute coronary syndromes. J Am Coll Cardiol. 1998;32:613-9.
9. van Elderen T, Maes S, Dusseldorp E. Coping with coronary heart disease: a longitudinal study. J Psychosom Res. 1999;47:175-83.

10. Mulder CL, Antoni MH, Duivenvoorden HJ, Kauffmann RH, Goodkin K. Active confrontational coping predicts decreased clinical progression over a one-year period in HIV-infected homosexual men. J Psychosom Res. 1995;39:957-65.

11. Carver CS, Pozo C, Harris SD, Noriega V, Scheier MF, Robinson DS, et al. How coping mediates the effect of optimism on distress: a study of women with early stage breast cancer. J Pers Soc Psychol. 1993;65:375-90.

12. Yu DSF, Thompson DR, Yu CM, Pedersen SS, Denollet J. Validating the Type D personality construct in Chinese patients with coronary heart disease. J Psychosom Res. 2010;69:111-8.

13. Yu XN, Zhang J, Liu X. Application of the Type D Scale (DS14) in Chinese coronary heart disease patients and healthy controls. J Psychosom Res. 2008;65:595-601.

14. Ou W, Liu T, Sun YJ, Sun YL. Quality of life and Type D personality in patients undergoing coronary artery stent implantation. J Clinic Rehab Tiss Eng Res. 2008;44:8635-8.

15. Bosworth HB, Siegler IC, Brummett BH, Barefoot JC, Williams RB, Clapp-Channing NE, et al. The association between self-rated health and mortality in a well-characterized sample of coronary artery disease patients. Med Care. 1999;37:1226-36.

16. Mommersteeg PMC, Denollet J, Spertus JA, Pedersen SS. Health status as a risk factor in cardiovascular disease: a systematic review of current evidence. Am Heart J. 2009;157:208-18.

17. Rideout E, Montemuro M. Hope, morale and adaptation in patients with chronic heart failure. J Adv Nurs. 1986;11:429-38.

18. Royal College of Physicians (Research Unit) and the British Geriatric Society. Standardised assessment scales for elderly people. London: Royal College of Physicians and British Geriatric Society; 1992.

19. Maier H, Smith J. Psychological predictors of mortality in old age. J Gerontol B Psychol Sci Soc Sci. 1999;54:44-54.

20. Gensini GG. A more meaningful scoring system for determining the severity of coronary heart disease. Am J Cardiol. 1983;51:606.

21. Denollet J. DS14: standard assessment of negative affectivity, social inhibition, and Type D personality. Psychosom Med. 2005;67:89-97.

22. Feifel H, Strack S, Nagy VT. Degree of life-threat and differential use of coping modes. J Psychosom Res. 1987;31:91-9.

23. Jiang Q, Shen X. In: Wang X, editor. Medical Coping Modes Questionnaire (MCMQ). Beijing: Chinese Journal of Mental Health Publishing House; 1999.

24. Brodman K, Erdmann Jr AJ, et al. The Cornell medical index: an adjunct to medical interview. J Am Med Assoc. 1949;140:530-4.

25. Weaver CA, Ko Y-H, Alexander ER, Pao Y-L, Ting N. The Cornell Medical Index as a predictor of health in a prospective cardiovascular study in Taiwan. Am J Epidemiol. 1980;111:113-24.

26. Lawton MP. The Philadelphia Geriatric Center Morale Scale: a revision. J Gerontol. 1975;30:85-9.

27. Hwang HL, Lin HS. Perceived enactment of autonomy and related factors among elders. J Nurs Res. 2003;11:277-86.

28. Cohen J. Statistical power analysis for the behavioral sciences. 2nd ed. Hillsdale: Lawrence Erlbaum Associates Publishers; 1998.

29. Preacher KJ, Hayes AF. SPSS and SAS procedures for estimating indirect effects in simple mediation models. Behav Res Meth Instrum Comput. 2004;36:717-31.

30. Martens EJ, Kupper N, Pedersen SS, Aquarius AE, Denollet J. Type-D personality is a stable taxonomy in post-MI patients over an 18-month period. J Psychosom Res. 2007;63:545-50.

31. de Jonge P, Denollet J, van Melle JP, Kuyper A, Honig A, Schene $\mathrm{AH}$, et al. Associations of type-D personality and depression with somatic health in myocardial infarction patients. J Psychosom Res. 2007;63:477-82. 
32. Pedersen SS, Denollet J. Validity of the Type D personality construct in Danish post-MI patients and healthy controls. J Psychosom Res. 2004;57:265-72.

33. Grande G, Jordan J, Kummel M, Struwe C, Schubmann R, Schulze F, et al. Evaluation of the German Type D Scale (DS14) and prevalence of the Type D personality pattern in cardiological and psychosomatic patients and healthy subjects. Psychother Psychosom Med Psychol. 2004;54:413-22.

34. Pedersen SS, Kupper N, Denollet J. Psychological factors and heart disease. In: Camm AJ, Lscher TF, Serruys WP, editors. The ESC Textbook of Cardiovascular Medicine. 2nd ed. Oxford: Oxford University Press; 2009. p. 1287-304.

35. Woo J, Ho SC, Wong EM. Depression is the predominant factor contributing to morale as measured by the Philadelphia Geriatric Morale Scale in elderly Chinese aged 70 years and over. Int $\mathbf{J}$ Geriatr Psychiatry. 2005;20:1052-9.
36. Sullivan MD. Maintaining good morale in old age. West J Med. 1997; 167:276-84.

37. Polman R, Borkoles E, Nicholls AR. Type D personality, stress, and symptoms of burnout: The influence of avoidance coping and social support. Br J Health Psychol. 2010;15:681-96.

38. Pereira DB, Antoni MH, Danielson A, Simon T, Efantis-Potter J, O'Sullivan MJ. Inhibited interpersonal coping style predicts poorer adherence to scheduled clinic visits in human immunodeficiency virus infected women at risk for cervical cancer. Ann Behav Med. 2004;28:195-202.

39. Kirkcaldy BD, Shephard RJ, Siefen RG. The relationship between physical activity and self-image and problem behaviour among adolescents. Soc Psychiatry Psychiatr Epidemiol. 2002;37:544-50.

40. Inouye J, Flannelly L, Flannelly KJ. The effectiveness of selfmanagement training for individuals with HIV/AIDS. J Assoc Nurses AIDS Care. 2001;12:73-84. 